\title{
General Theory of Harmonics Generation Through Energy Transformation
}

\author{
Don E. Czyzyk ${ }^{1}$ \\ ${ }^{1}$ Sri Int., Menlo Park, Calif., USA \\ Correspondence: Don E. Czyzyk, Sri Int., 333 Ravenswood Ave, Menlo Park 94025, Calif., USA. E-mail: \\ don.czyzyk@sonic.net
}

Received: March 10, 2014 Accepted: March 25, 2014 Online Published: April 4, 2014

doi:10.5539/apr.v6n3p8

URL: http://dx.doi.org/10.5539/apr.v6n3p8

\begin{abstract}
Energy increases the vibrations of the medium absorbing it. Energy, whatever its form, when detected by the appropriate sensor, can be converted into a signal composed of moving electric charges. We found, amazingly, that this signal is composed, somehow, of a continuum of sinusoidal frequencies called a Harmonic Spectrum $(\mathrm{H} / \mathrm{S})$. Subsequently numerous signal generating experiments, utilizing mechanical, acoustic, pneumatic, thermal, magnetic, hydraulic, photonic devices and batteries as energy sources, were conducted to explore harmonics generation. Ultimately the aim of this research was to determine, in these cases, the fundamental origin of harmonics generation.

This research paper is preliminarily an experimental investigation into the nature of harmonic generation. The empirical data provided was gathered from experimental results and was used to develop a theoretical model to explain how energy is transformed into an H/S. Thus we find that conduction electrons, through the ongoing intricate interplay of processes involving acceleration, constructive/destructive interference (superposition), and Sympathetic Resonance (S/R), are intimately involved in the generation of harmonics. This combination of factors seems to be a principal means by which harmonics are generated, but these factors should not be thought applicable only to electronic circuits.
\end{abstract}

To our knowledge this paper, at present, provides the only theoretical model that fundamentally addresses the questions of how and why harmonics are generated.

Keywords: energy, signal envelope, conduction electrons, acceleration, constructive/destructive interference (superposition), sympathetic resonance, harmonics

\section{Introduction}

The study of how and why things vibrate was first undertaken at approximately $3000 \mathrm{BC}$ and continued up until the late 19th century (de Silva, 2000). But at the start of the 20th century until the present, the primary focus changed into finding applications for the knowledge gained about the nature of vibrations. Although this subject has been, in general, extensively studied by many researchers (de Silva, 2000), one aspect that has received little attention is how, at the electron level, frequencies (Harmonics) are generated. The current degree of understanding about harmonic generation is limited to what is the envelope (shape) of a signal, the H/S content, the frequency amplitude and phase. There is currently no theoretical model or mechanism available that provides an understanding of the nature of harmonic generation in terms of the behavior of electrical charges. This is because experimental researches into the nature of harmonics at that time were technologically limited in being able to delve deeply into understanding the nature of harmonics from an atomic perspective compared to the capabilities, abilities and understanding we at present posses. So efforts to more deeply understand the nature of harmonics were greatly curtailed.

Then because they were not technologically advanced enough eventually their efforts and focus changed to finding practical applications of the knowledge gained about harmonics. Along with the change of focus came the use of several mathematical methods that attempted to provide understanding, such as the Fourier analysis, but they only described harmonic behavior, not how and why they are generated or the mechanisms behind it. Without a theoretical framework and explanation, all these facts have limited significance. It is this dearth that served as the motivation to perform this study, which aims to aid in revealing the nature of this important 


\section{phenomenon.}

Through extensive experimentation, we found that the envelope of a signal, whether a segment of a pulse, a whole pulse, or a train of pulses, is composed of a unique continuum of frequencies called an H/S. The H/S is the result of conduction electrons absorbing the energy of the pulses and transforming them into a continuum of frequencies. This paper is important because it disseminates the actual functioning of en masse electrons (electric charges) beyond what is currently realized. Fundamental knowledge of how electrons really behave will help us to better understand the different areas where harmonics occur and may have application in other areas as well.

\section{Methods and Materials}

We began this research with the desire to determine the participants involved in the generation of harmonics. To accomplish this, we started with the circuits in Diagram 1, which aided us in determining what entities were involved in harmonic generation. We then used the circuit in Diagram 2 to generate all the individual segments that comprised the following one-shot pulses: sine wave, triangle, sawtooth, and square waves. Whenever necessary, we used the circuit in Diagram 3 to determine the phase of a pulse segment. We then examined each pulse segment with a Hewlett-Packard audio frequency spectrum analyzer to observe their $\mathrm{H} / \mathrm{S}$ because all signals analyzed were in the audio frequency range.

The study started by gradually generating a one-shot pulse by adding the appropriate segments Figures $1 \mathrm{~A}$ and 1B, and then continued to add completed pulses to this initial pulse until three pulses were formed Figures 1 D-1F. These three pulses combined to form the beginning of a pulse train. We could have added more pulses, but only two or three pulses are needed to begin to develop the harmonic pattern that is characteristic of that particular signal envelope. The same basic procedure was used to examine sine wave, triangle, and sawtooth pulses as well. In addition, we made videos of all our results to be able to examine, in detail, how all the H/Ss contribute to the formation of the final characteristic $\mathrm{H} / \mathrm{S}$.
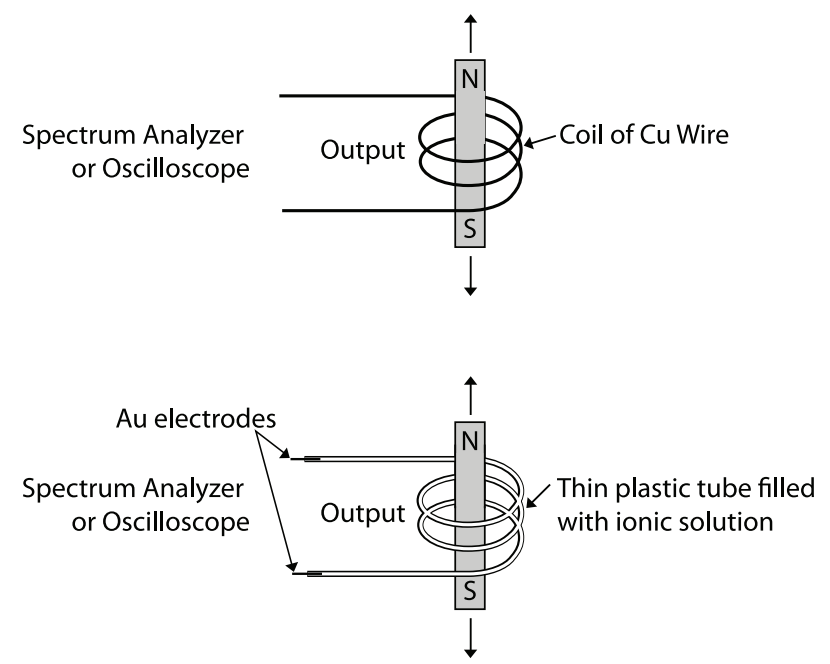

Diagram 1. These two different circuits exemplify how electrons or ions are made to flow by the moving magnetic field “Ampere's Law" (Sears \& Zemansky, 1970). Signals generated by these circuits were found to contain harmonic spectrums. The wire circuit used a Copper $(\mathrm{Cu})$ coil and the plastic tube coil was filled with a low resistance electrolyte and each end was connected with gold $(\mathrm{Au})$ electrodes 


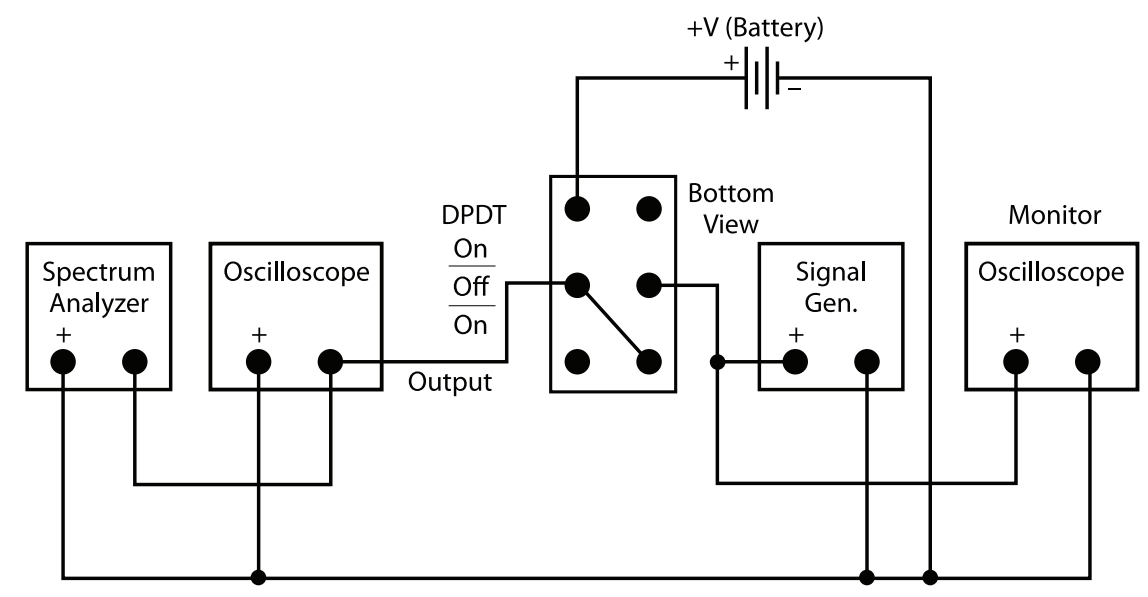

Diagram 2. With this circuit, using a combination of manual and electrical methods generated various low frequency signals made from either segments of a pulse, a complete pulse, or a signal made of a train of pulses. These signals, when generated, were simultaneously monitored and analyzed by the appropriate test instruments within this circuit setup. This setup includes an audio frequency spectrum analyzer, oscilloscope, audio signal generator and a manually operated Double Pole Double Throw (DPDT) switch
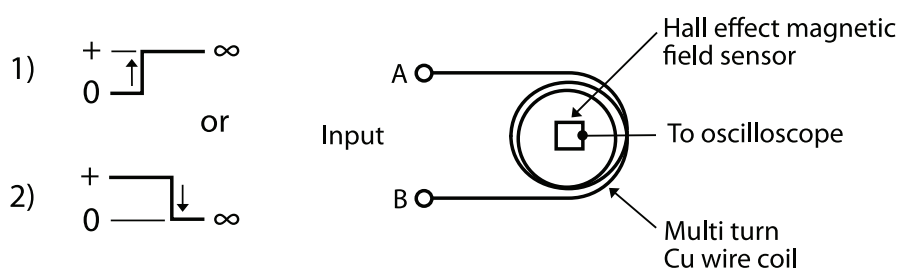

Diagram 3. The flow of the conduction electrons in the $\mathrm{Cu}$ wire coil will generate a magnetic field; next, depending on the direction of the current flow, this flow will determine the orientation of the magnetic field produced by the coil Ampere's Law (Sears \& Zemansky, 1970). With this information, phase comparisons between pulse segments can be determined

\section{Results}

Using the circuit in Diagram 1, it was observed that harmonics are initially generated because of the en masse movement of conduction electrons in response to absorbing the energy from, in this case, a moving magnet. But with the proper sensor other energy sources can be utilized whether they come from mechanical, acoustic, pneumatic, thermal, hydraulic, photonic devices as well as other magnetic sources.

Next, we used the circuit in Diagram 2 to generate low frequency pulses and to isolate segments of a one-shot pulse that were of interest, such as the rising segment $A \uparrow$ and the segment $\vec{B}$, falling segment $C \downarrow$ as seen in Figure $1 \mathrm{C}$ which shows the resultant harmonic series when these segments are combined to make a completed one-shot pulse.

All pulse segments generate a transient H/S. Now because the H/S of any one-shot pulse or pulse segment is seen to quickly rise to a maximum and then decay to zero, all of the graphs in this paper show only an approximation of what the signal's H/S looked like when it reached its maximum. In addition, there will be less harmonics displayed in the "Frequency Domain" graphs of all complete 1-shot pulses and they typically will have a range of amplitudes different from those found in pulse segments. 
1 A

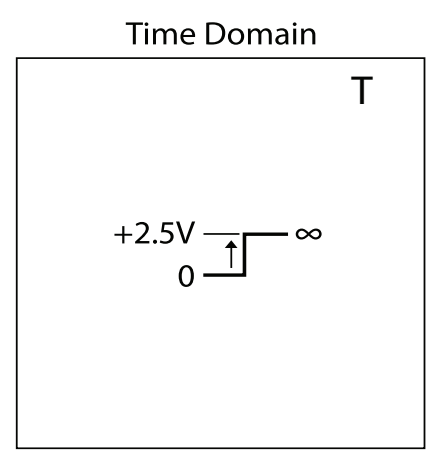

$1 B$

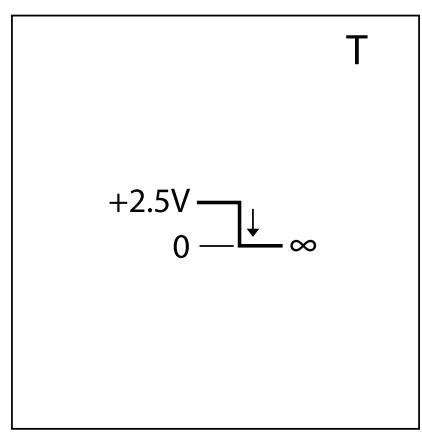

1C

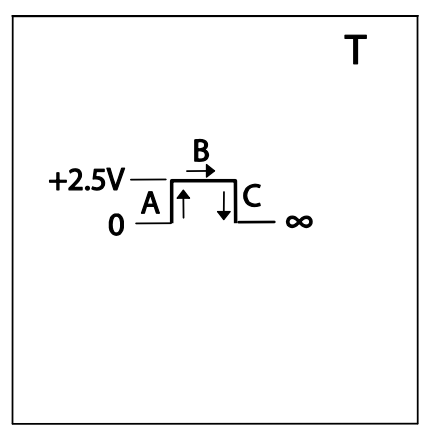

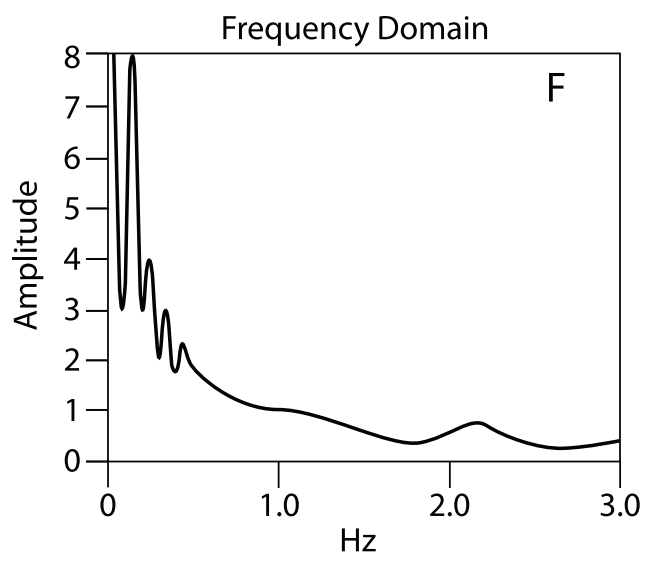
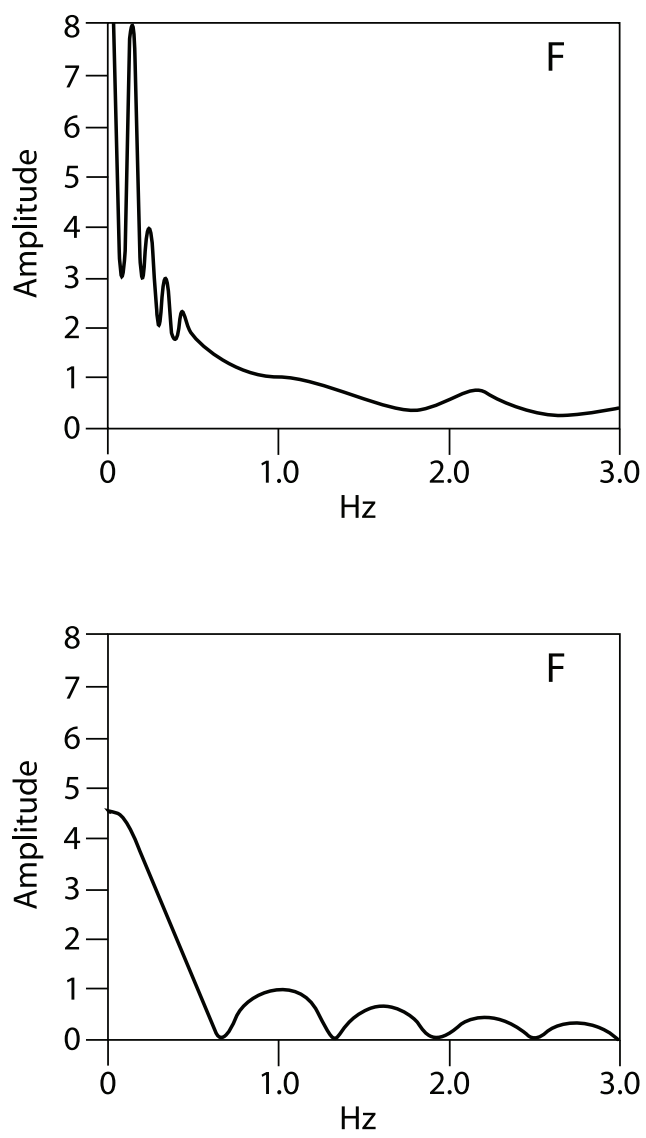

Figure 1 (A-C). The segment envelopes in the Figures 1A and 1B graphs combine to produce the one-shot pulse in graph Figure $1 \mathrm{C}$. The harmonics generated by the segments $A \vec{A}, \vec{B}$ and $C \downarrow$ will interact causing some harmonics to be increased and some to be reduced or eliminated because they are out of phase with each other.

The result of this interaction process is Figure $1 \mathrm{C}$ and the generation of a unique $\mathrm{H} / \mathrm{S}$

Starting with the signal pulse in Figure 1D then we added a second pulse Figure $1 \mathrm{E}$ and finally a third pulse Figure 1F. By combining these three pulses, it can be seen how the pattern gradually changes to form the harmonic pattern characteristic of a signal called a square wave with a 50\% duty-cycle. As previously mentioned, we could have added additional pulses, but we found that it only requires two or three pulses to generate the basic harmonic pattern that is indicative of the $\mathrm{H} / \mathrm{S}$ made by a train of pulses. 
$1 D$
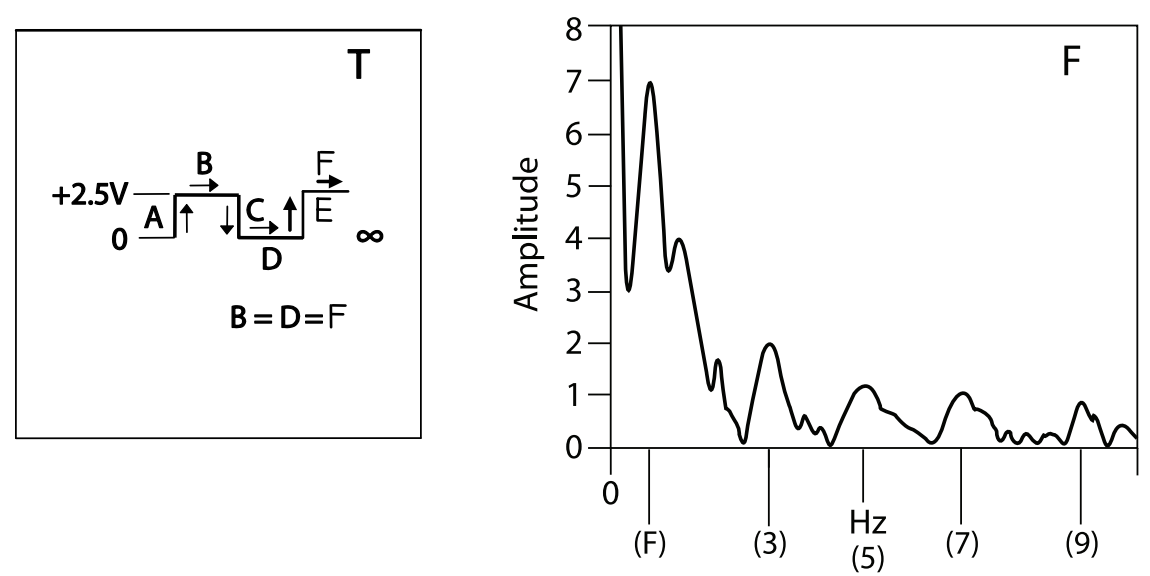

$1 \mathrm{E}$
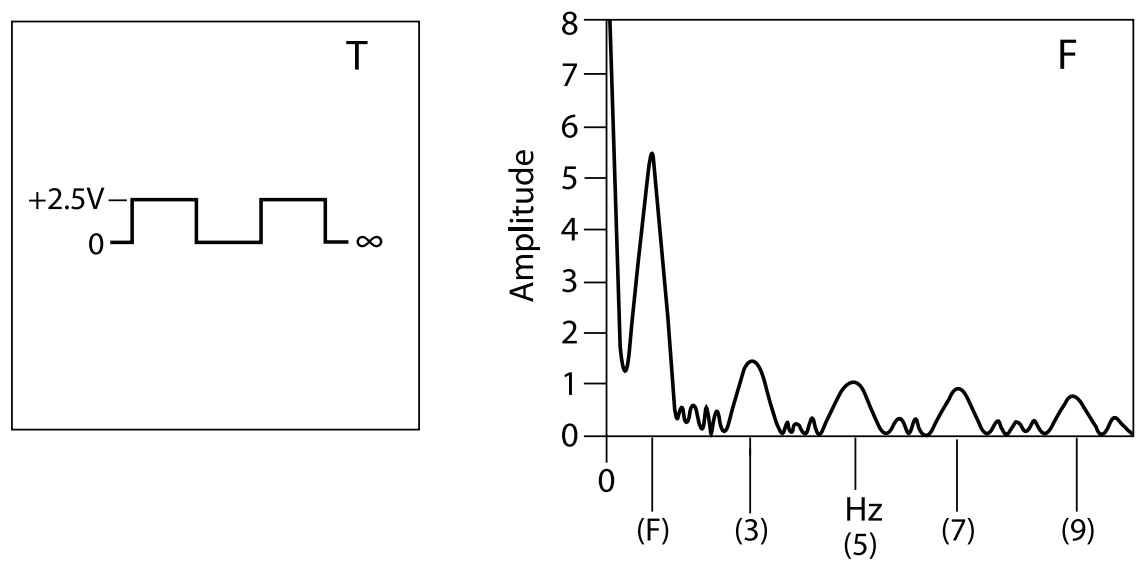

$1 \mathrm{~F}$
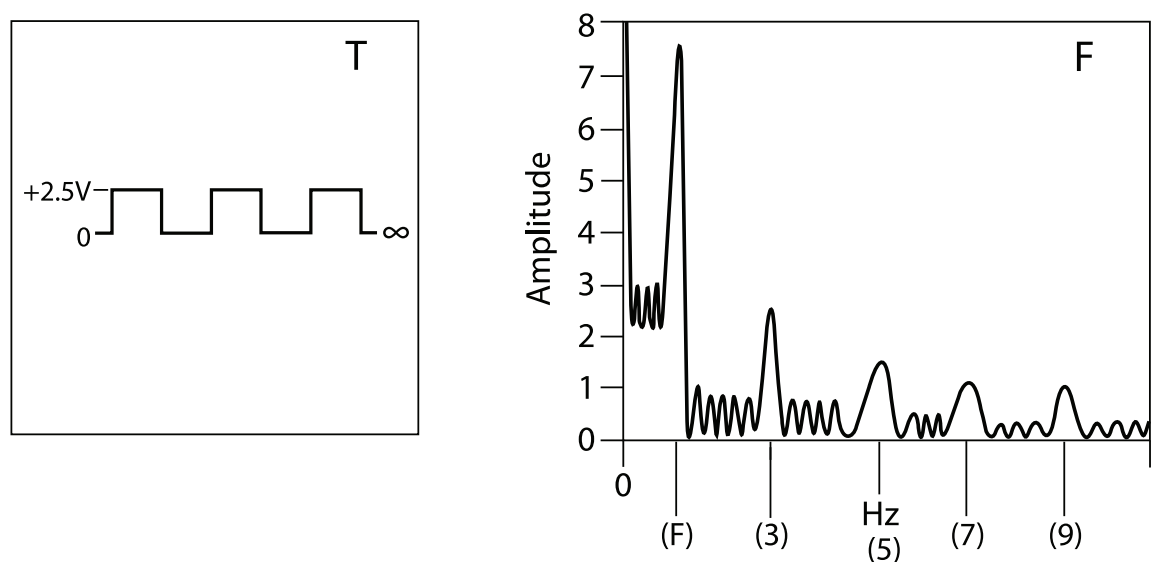

Figure 1 (D-F). This collection of pulse envelopes show the contribution of each added pulse to the resulting H/S. This H/S has only odd harmonics which are characteristic of a square wave with a $50 \%$ duty cycle

In order to observe the effect of varying the width of the envelope of a one-shot pulse and its resulting $\mathrm{H} / \mathrm{S}$ we started with the narrow pulse in Figure 2A then, while keeping the amplitudes constant, increased the width of the next pulse to that in Figure 2B and further increased the width of the final pulse to that in Figure 2C. 
2A
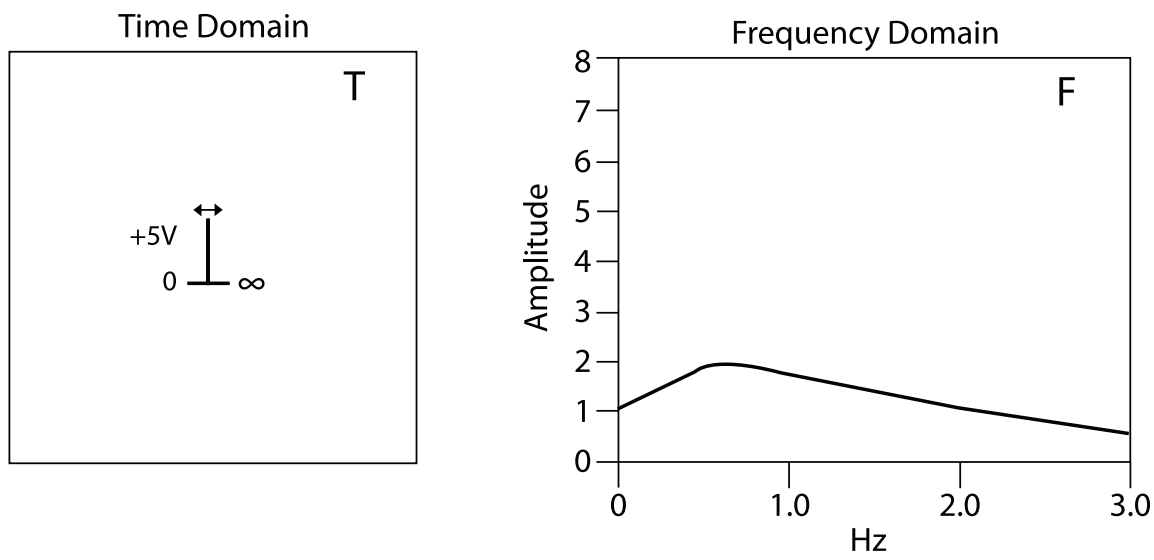

2B
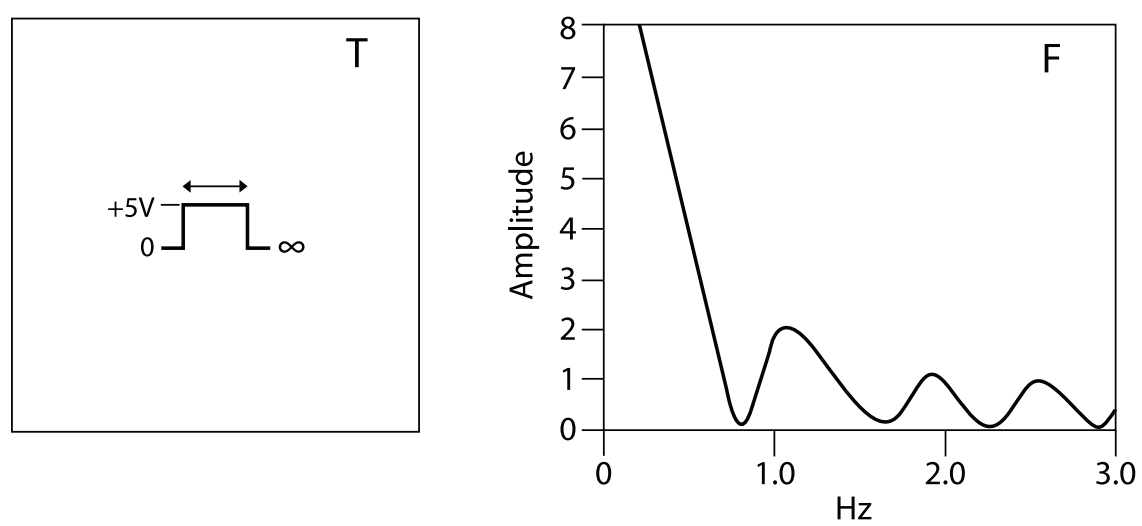

2C
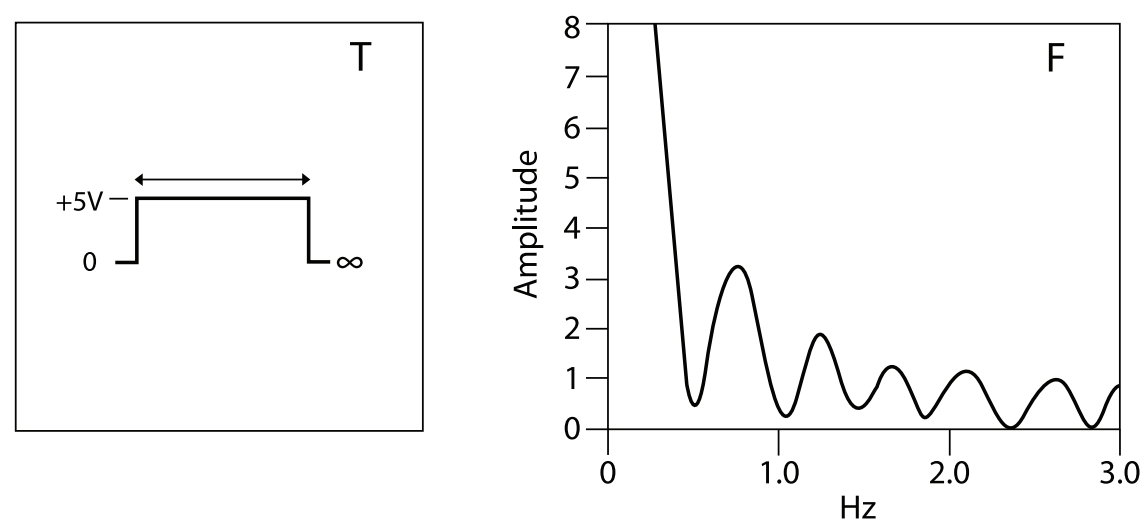

Figure 2 (A-C). When only the width of these one-shot pulses is changed, this causes a change in their respective $\mathrm{H} / \mathrm{S}$

Another type of signal that we investigated was composed of two one-shot pulses, whose duty cycle was changed from $25 \%$ to $50 \%$ and then to $75 \%$. These changes resulted in the H/S seen in Figures $2 \mathrm{D}-\mathrm{F}$. 
2D
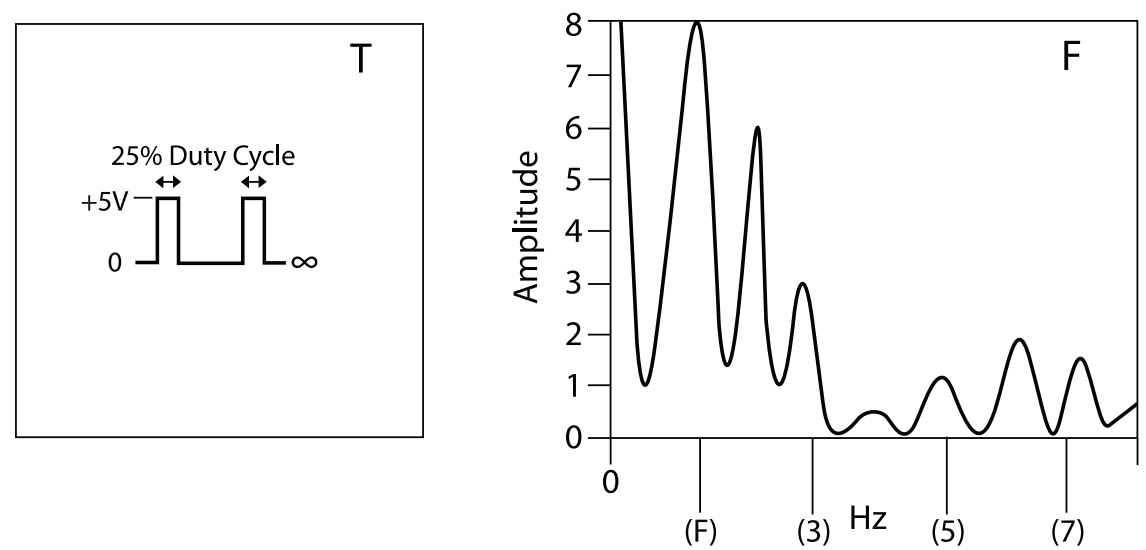

$2 \mathbf{E}$
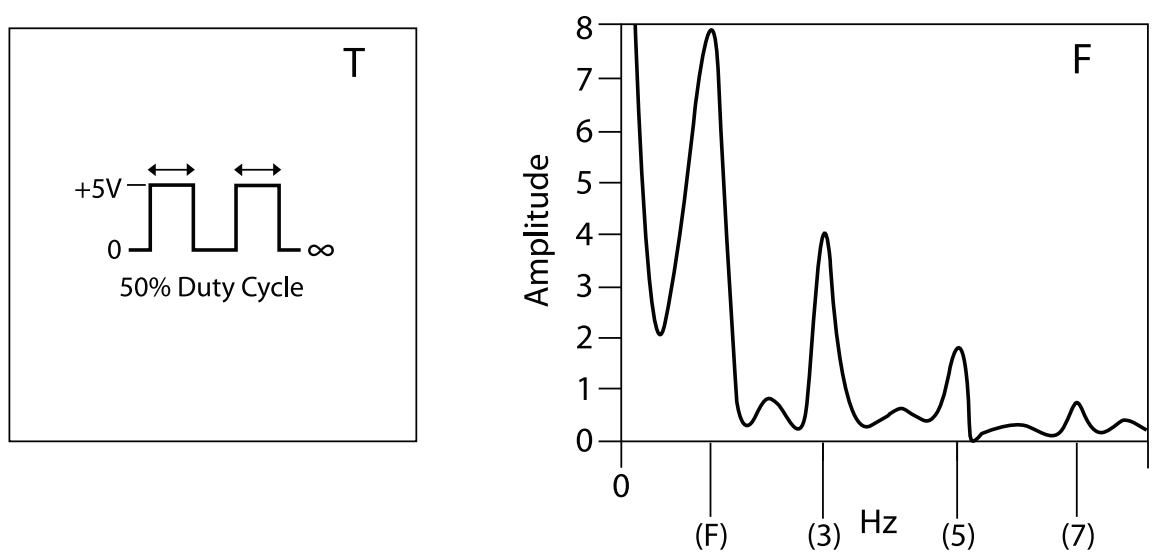

2F
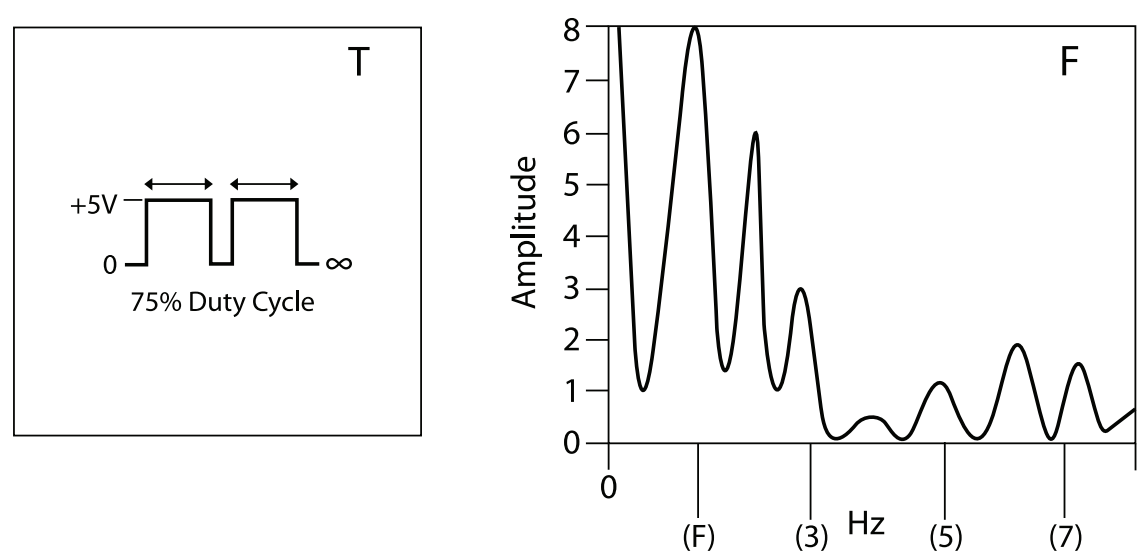

Figure 2 (D-F). These graphs show what happens to the H/S when a signal composed of two or more one-shot pulses changes from a $25 \%$ to $50 \%$ duty cycle and then to a $75 \%$ duty cycle. Note that the $\mathrm{H} / \mathrm{S}$ of the $25 \%$ and the $75 \%$ duty cycle envelopes are identical

The final signal envelopes we examined were one-shot signals of a sawtooth, triangle, and sine wave. The sawtooth is comprised of the segments shown in Figures 3A and 3B, and when these segments are combined, they create the signal envelope in Figure 3C. The triangle in Figure 3F is made by combining the segments in Figures 3D and 3E. Using a spectrum analyzer it was determined that the segments in Figures 3D and 3E have an identical H/S. Then, using the circuit in Diagram 3, we found that these two pulse segments were also out of phase with each other. The same was true for the segments in Figure 3G and $3 \mathrm{H}$ which form the sine wave in Figure 3I. 
3A
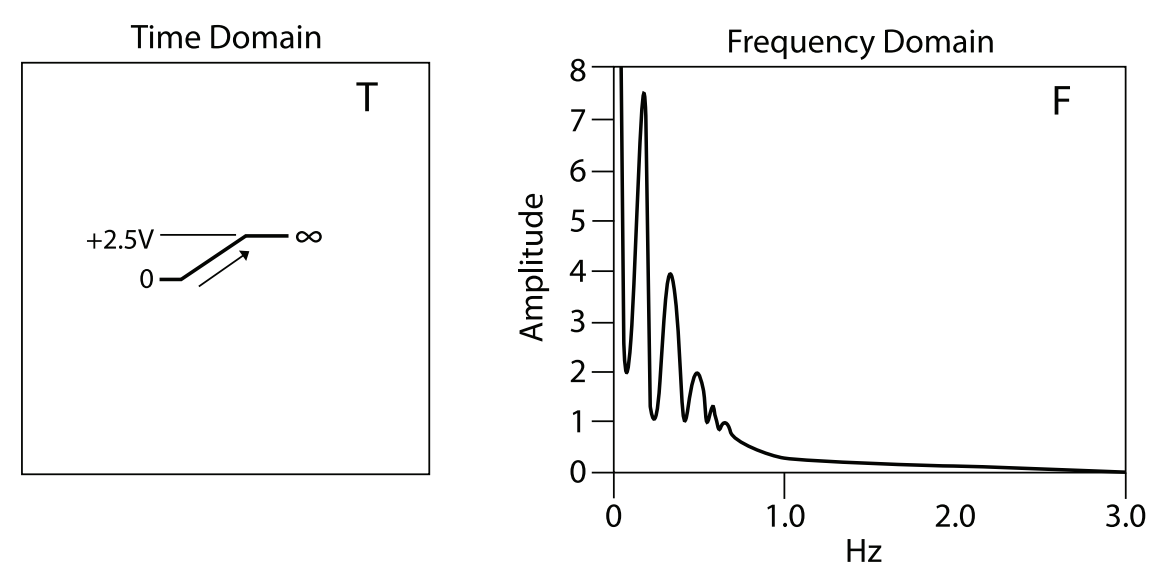

3B
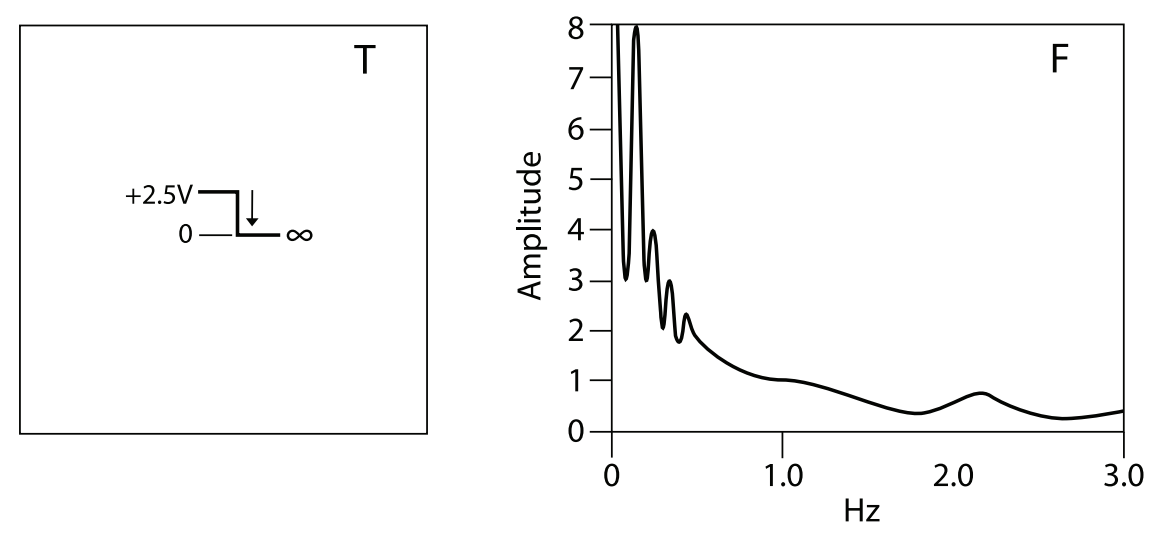

$3 \mathrm{C}$
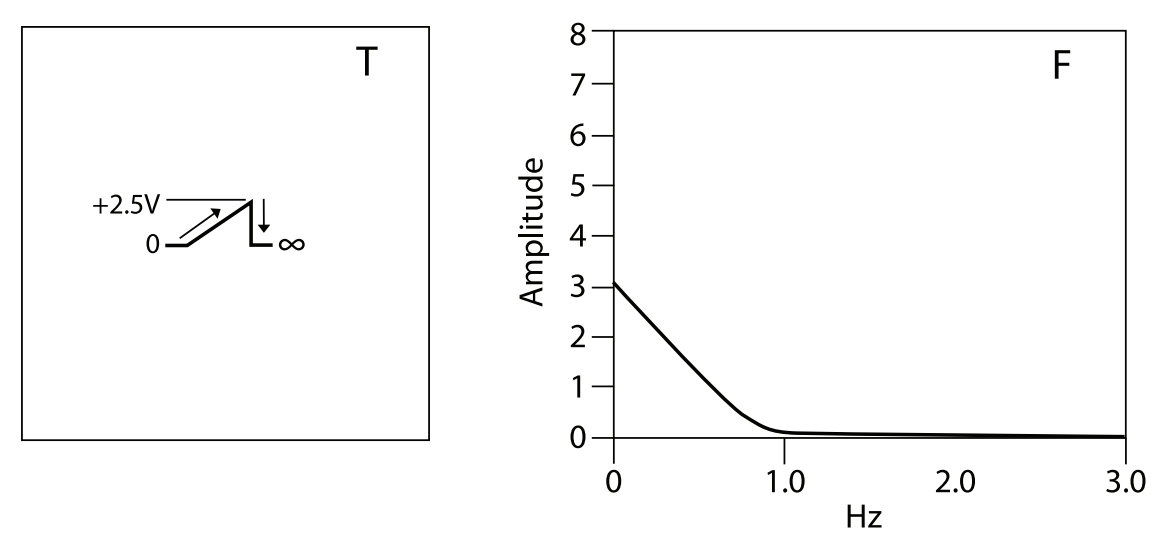

Figures 3 (A-C). These examples show pulse segment in Figure 3A with a slowly rising slope and another pulse segment in Figure 3B with a slope that abruptly drops to zero. When these segments are combined, a one-shot sawtooth pulse is produced as seen in Figure 3C 
3D
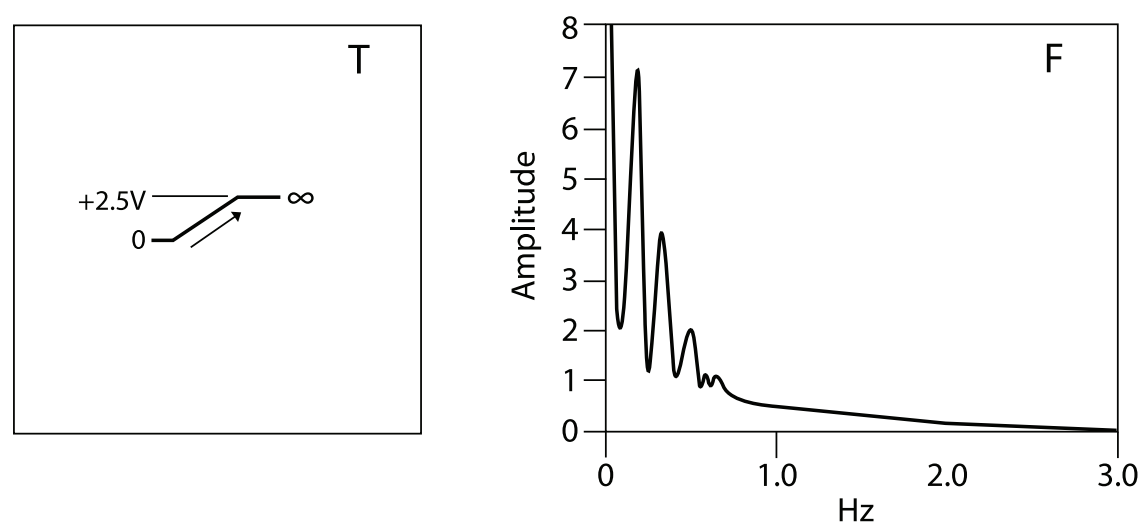

$3 \mathrm{E}$
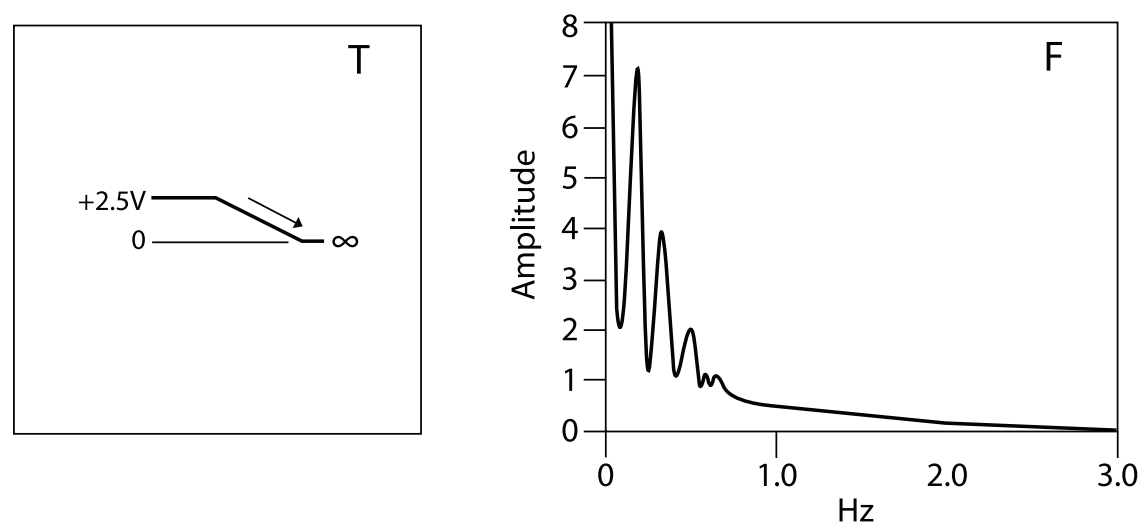

$3 \mathbf{F}$
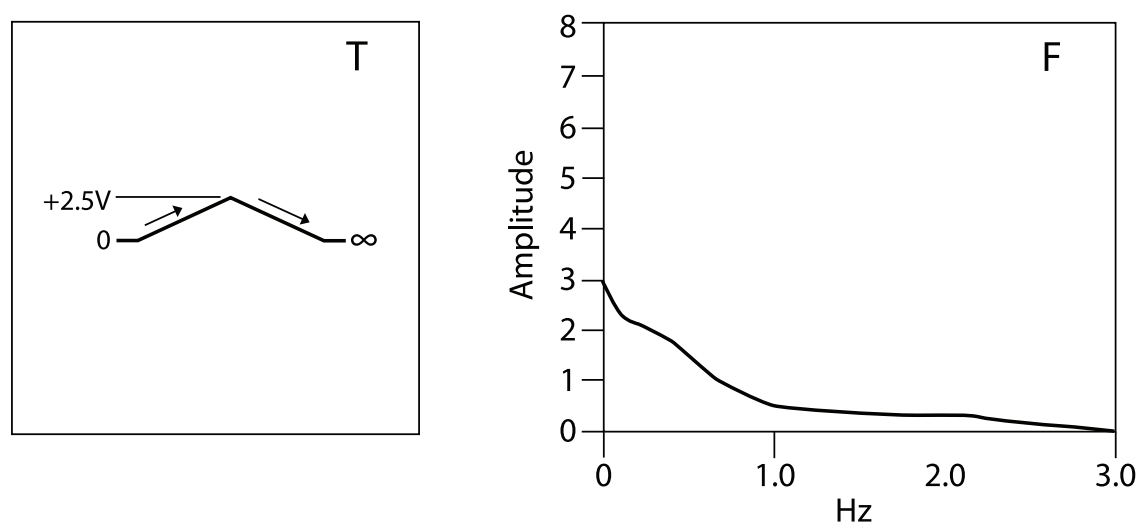

Figures 3 (D-F). In these examples, the one-shot triangle pulse in Figure 3F is the result of combining two segments, one segment that has a slowly rising slope in Figure 3D and the other segment a slowly declining slope in Figure 3E. Because these slopes are moving in opposite directions this results in their respective H/S being out of phase with each other 
$3 G$
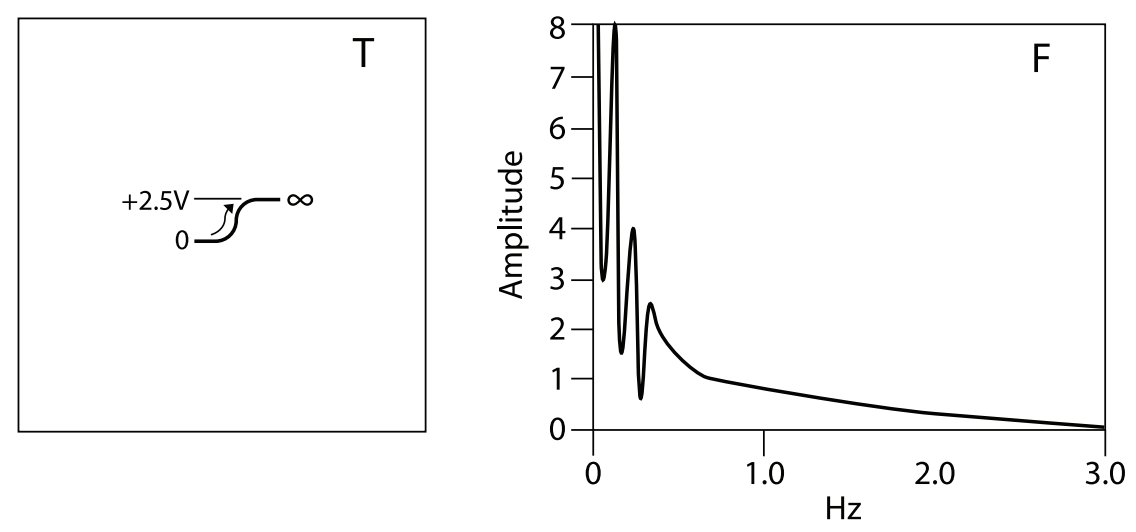

3H
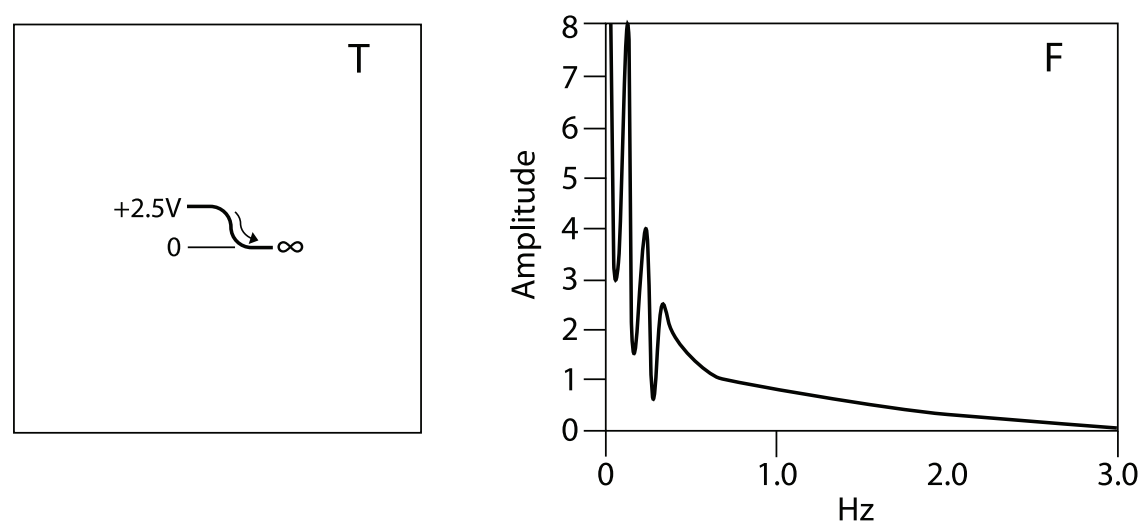

3I
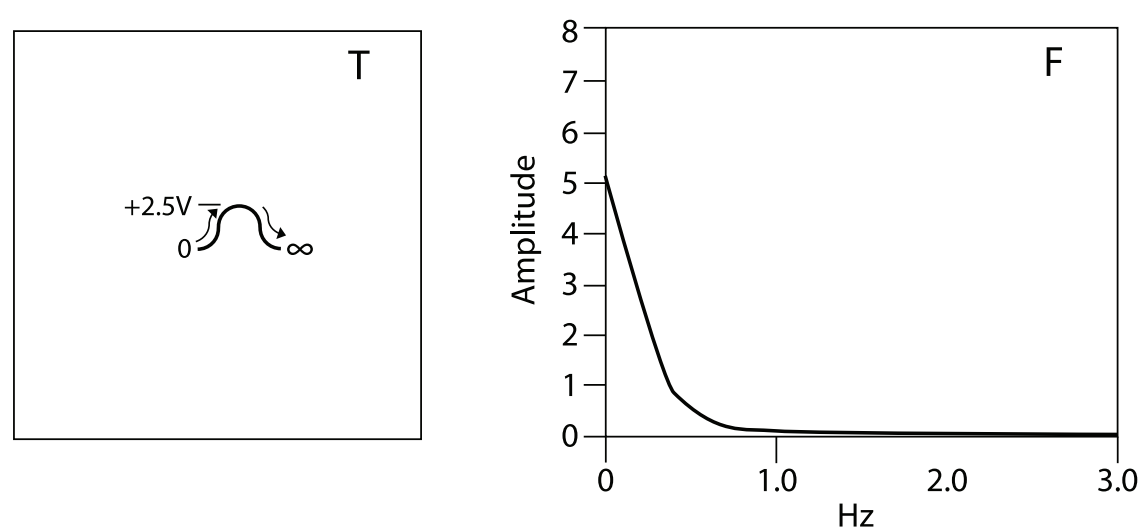

Figures $3(\mathrm{G}-\mathrm{I})$. These graphs show the gradually rising segment in Figure $3 \mathrm{G}$ and the declining segment in

Figure $3 \mathrm{H}$ that, when combined, create the one-shot sine wave pulse in Figure 3I and its $\mathrm{H} / \mathrm{S}$. Also since the segment in Figure 3G is raising and the segment in Figure 3H is declining this will result in their H/S's being out of phase as well

\section{Conclusion}

This research is primarily an experimental investigation into the nature of harmonic generation via the en masse behavior of electric charges in general and conduction electrons in particular. The following example describes a simple but thought-provoking electronic circuit that can generate harmonics. This circuit consists of a battery connected in series with a switch that when turned on and off in a periodic pattern will generate an $\mathrm{H} / \mathrm{S}$ that is composed of a continuum of frequencies. It is at this point that one may begin to wonder how is it possible that, 
by simply turning a switch on/off that this generates a harmonic spectrum and additionally one may ask what is the origin and mechanism responsible for the generation of these harmonics.

For this example, as well as others, this paper provides a theoretical model to fill the void that now exists when it comes to explaining how and why these harmonics are created. Because this investigation has been primarily experimental it still leaves this subject, the en masse behavior of electric charges, open to further mathematical analysis of by those so inclined.

The answers to the following questions will support the theoretical model proposed in this paper.

- Are electric charges, i.e., conduction electrons, involved in harmonic generation?

- What specifically must be done to conduction electrons for them to generate harmonics?

- How is the harmonic spectrum, further modified when the energy of additional pulse segments or pulses are absorbed by conduction electrons?

The answer to the first question was attained from the results gathered using the circuits in Diagram 1. These are simple but effective circuits similar to those used by Michael Faraday in his investigation of the interactions between electric charges and magnetic fields (Faraday, 1831). The first circuit shows that the electrons in the coil of copper wire will be moved, that is, be made to flow when the coil is cut by the magnetic lines of force from a moving bar magnetic. If this electron flow is analyzed with a spectrum analyzer, it will be seen that an $\mathrm{H} / \mathrm{S}$ has been generated.

Next, we used a coil made of thin plastic tubing that was filled with a low-resistance electrolyte and used gold $\mathrm{Au})$ wire as electrodes; this tubing setup was tested in the same way as the coil of wire. The results of these two tests showed as long as there are movable electric charges, in this case electrons/ions, they will vibrate when they absorb energy and in so doing generate an electric signal. When analyzed, it will be seen that this signal also has lower energy consequently the harmonics in the H/S will have lower amplitudes because ions are more massive and so are slower than electrons. Thus these tests show that only the movement of electric charges such as electrons or ions was observed to produce harmonics.

The answer to the second question was partially given in the answer to the first. The partial answer was that conduction electrons have to be made to move but more specifically by accelerating them and what is also important here is the degree of acceleration. When conduction electrons absorb the energy of a signal they transform that energy into an H/S. The degree of acceleration is determined by the angle of slope of a signal. For example Figure 1C shows a pulse segment whose slope has a large angle A $\uparrow$ while in Figure $3 \mathrm{~A}$ the segment shows a slope $\rightarrow$ with a smaller angle and Figure $1 \mathrm{C}$ also shows segment $\overrightarrow{\mathrm{B}}$ with zero slope. The angle of slope in these examples determines the amount of energy with which conduction electrons will be accelerated. The effect due to generating pulse signals that contain slopes with different angles is that at higher slope angles more energy is provided to the conduction electrons thereby causing these electrons to generate a larger series of sinusoidal vibrations (Harmonics). This result can be seen in all the graphics contained in Figures 1, 2, and 3.

An important point to remember here is that these harmonic frequencies are independent of the natural vibrations produced, at room temperature, in the conductors used in electronic circuits.

The first two questions were of a general nature; thus, we chose to use brevity when answering them. However, the answer to the third question will begin with the pulse shown in Figure 1D and will now be explained in more detail. In Figure 1D, each segment is expressed as playing a unique part in the transformation of energy into harmonic frequencies. The pulse segments $\mathrm{A} \uparrow$ and $\mathrm{E} \uparrow$ (rising edge) and $\mathrm{C} \downarrow$ (declining edge) are segments where abrupt acceleration was occurring and where harmonics were generated. When the segment $\mathrm{A} \uparrow$ (rising edge) transient signal, is applied to an electric circuit, its energy is absorbed by conduction electrons, which generates an initial harmonic series. This series is a continuum of sine waves ranging from very low to very high frequencies. The harmonics generated go through phase changes over time until their energy has dissipated.

The next segment B determines the width of the pulse and thus indicates the duration of the pulse. The importance of these types of segments is that they indicate time duration, and that will determine what the phases will be of the harmonic frequencies that were generated by the initial pulse segment $\mathrm{A}$. When the segment $\mathrm{C} \downarrow$ occurs, it causes the generation of another H/S that is similar to that seen in Af. The segment $\mathrm{C} \downarrow$, is also transient and occurs because the pulse voltage has decreased to zero, causing an electron current flow that is in the opposite direction to that of the flow initiated by segment A4. This reversed current flow is produced because electrons were held at the voltage potential produced by the segment $\mathrm{A} \uparrow$ and the potential was kept constant for the duration of segment $\vec{B}$. However, when the voltage potential drops to zero volts, such as in the segment $\mathrm{C} \downarrow$ This allows the electrons to flow in the opposite direction because of Ampere's Law (Sears \& Zemansky, 1970). 
Then, because of either constructive or destructive interference (superposition), there will be amplification or attenuation of harmonics because the phases of some of the harmonics generated initially by the pulse segment $\mathrm{A} \uparrow$ are in or out of phase with the harmonics generated by the electron flow due to segment $\mathrm{C} \downarrow$.

Due to the superposition process all the graphs showing the H/S of complete 1-shot pulses will show them to have different harmonics and amplitudes in their $\mathrm{H} / \mathrm{S}$ because there will be reduction or elimination of some harmonics due to some frequencies being out of phase with others.

The joining of the segments $A \uparrow, \vec{B}$ and $C \downarrow$ forms the single pulse seen in Figure 1C. But because this is only a single pulse envelope it does not constitute a complete wavelength so it won't be able to cause conduction electrons to generate a meaningful $\mathrm{H} / \mathrm{S}$. Yet, although this is only a single pulse, it will still be able to produce a crude harmonic series, but this series will not have a well-defined fundamental frequency, only a small collection of odd and even harmonic frequencies of low amplitude. To make the pulse signal in Figure 1C able to begin to create a meaningful H/S, we need to add the segments $\mathrm{D} \rightarrow, \mathrm{E} \boldsymbol{\uparrow}$ and $\overrightarrow{\mathrm{F}}$ as shown in Figure 1D.

With the segments D, E and F added, we now have a signal whose envelope is one complete wavelength plus just the beginning of the second pulse. This signal will energize the conduction electrons causing them to begin to resonate en masse due to sympathetic resonance at this wavelength. Sympathetic Resonance $S / R$ is an effect that occurs where by a body will vibrate at the same frequency as that of a nearby vibrating body by absorbing its vibrational energy. A classic example of this is causing a tube to vibrate by the application, with a speaker, of a frequency that will make it resonate. We used a variation of this example to observe how a tube begins to vibrate/resonate as it gradually absorbs the energy of a pulse applied one pulse at a time. By conducting this experiment we observed that this caused the amplitude of the tubes vibration to gradually increase with each pulse and if enough pulses were applied this increased the amplitude to a maximum and the maximum remained constant even if more pulses were added. This pattern is observed in cases where the phenomenon of resonance occurs. Similarly when the energy of a train of pulses is absorbed by conduction electrons it will cause these electrons to vibrate/resonate at all the harmonic frequencies contained in its $\mathrm{H} / \mathrm{S}$ due to $\mathrm{S} / \mathrm{R}$.

Again the important point here is to remember that these harmonic frequencies are independent from the naturally occurring frequencies (vibrations) of the conduction electrons in a wire at room temperature.

The harmonic series produced by the pulse seen in Figure 1D will contain the beginnings of a fundamental frequency and its multiples. However, because this harmonic series is the result of a single pulse of a whole wavelength, it produces an $\mathrm{H} / \mathrm{S}$ whose harmonics have low amplitudes. Adding more of these pulses will produce an $\mathrm{H} / \mathrm{S}$ that will have more energy, thus producing a better defined collection of larger harmonics starting with a fundamental frequency and, in this case, only odd harmonics, as seen in Figures 1D-1F.

It can now be understood how conduction electrons can generate a continuum of sinusoidal frequencies. Because when conduction electrons become energized by absorbing the energy of a signal with a periodic waveform they have the ability to transform, as shown in this paper, the energy of that signal into a collection of odd and even sinusoidal frequencies (harmonics) that are multiples of the fundamental frequency of that signal. The resultant harmonic series produced is characteristic of the harmonic content contained within the envelope of the signal.

Knowing how and why the transformation of energy, by electric charges such as conduction electrons, into a harmonic series of frequencies occurs is important because this phenomenon and its variations occur in numerous technological and scientific disciplines.

Some examples of where this conduction electron behavior may be occurring is in the process of hearing, where the energy of a sound wave is transformed into an electrical signal and then processed by the brain into what we perceive as sound. Another example is how a change in the physical activity of the human body is detected and converted into electrical signals by the nervous system: If applied to the heart, these signals will control the rhythmic rate of the heartbeat. Finally, another example is how light energy, an electromagnetic wave, is detected and converted by the human eye into electrical signals and then routed into the brain to be processed into a perceived visual image.

For those who may want to do further research in this area of harmonics be aware that actuators such as speakers, solenoids and relays and sensors like microphones and piezoelectric devices were unable to convert, with acceptable fidelity, various electrical signals into equivalent mechanical signals and vice versa except if these devices are operated at low audio frequencies. Because of this we were limited to performing low frequency experiments but this could be remedied with the development of linear higher frequency actuators/sensors.

Also a useful addition to this research, had we the means, would have been the use of computer simulation to investigate the en masse behavior patterns of electric charges due to modulation by signals from an outside 
energy source. A future area of research would benefit from using computer simulation to further investigate this important phenomenon.

There have been articles written describing methods by which multi-harmonics can be generated by use of acousto-electric (Beliaev, 1962), conduction electrons (Denisenko, 1978), microwave (Karatzas, 2009), electrojets (Kuo, 2002) and optical (laser) signals (Renk, 2012). These methods involve the behavior of electric charges; however none of these methods provide a theoretical model by which harmonics are generated by the en masse flow of electric charges. There are undoubtedly other areas where this important phenomenon occurs and where a deeper understanding of the mechanism of harmonic generation will open many other doors for further exploration.

\section{References}

Beliaev, L. M. (1962). Amplification of Acoustic Waves through Interaction with Conduction Electrons. Phys. Rev., 127, 1084-1090. (Ref. 5) Acousto-Electric Effect The Great Soviet Encyclopedia (3rd ed.), (1970-1979).

de Silva, C. W. (2000). History of Vibration. CRC Press LLC.

Faraday, M. (1831). Experimental Researches in Electricity. Great books of the western world (Vol. 1). Book \#45, 1990.

Fil, V. D., Gaiduk, A. L., \& Denisenko, V. I. (1978). Nonlinear Acoustic Properties of Conduction Electrons. Journal de Physique Colloques, 39(C6), 543-544. http://dx.doi.org/10.1051/jphyscol:19786244

Karatzas, N. E., \& Georges, A. T. (2009). Model for ultrafast harmonic generation from a gold surface: extraction of dephasing times for continuum-continuum transitions. Journal of the Optical Society of America B, 26(12), 2218-2227. http://dx.doi.org/10.1364/JOSAB.26.002218

Kuo, S. P. (2002). Generation of ELF and VLF radiation by ionospheric electrojet modulation using high frequency heating waves. Plasma Science, ICOPS 2002. IEEE Conference Record - Abstracts. The 29th IEEE International Conference on. http://dx.doi.org/10.1109/PLASMA.2002.1030439

Renk, K. F. (2012). Basics of Laser Physics: For Students of Science and Engineering. Springer.

Sears, F. W., \& Zemansky, M. W. (1970). Ampere's Law. University Physics (4th ed., Chapter 32).

\section{Copyrights}

Copyright for this article is retained by the author(s), with first publication rights granted to the journal.

This is an open-access article distributed under the terms and conditions of the Creative Commons Attribution license (http://creativecommons.org/licenses/by/3.0/). 